\title{
Reflections of the Angiotensin Receptor Blocker Recall by the FDA and Repercussions on Healthcare
}

\author{
Michael Gillette $^{1} \cdot$ Addison Taylor $^{1} \cdot$ Djenita Butulija $^{1} \cdot$ Himabindu Kadiyala $^{1} \cdot$ Hani Jneid ${ }^{1}$ \\ Published online: 21 April 2020 \\ (C) Springer Science+Business Media, LLC, part of Springer Nature 2020
}

\begin{abstract}
Purpose Beginning in July of 2018, the FDA issued a voluntary recall regarding the presence of a contaminant found in the manufacturing of valsartan. What would ensue has become a largely unprecedented sequence of alarming events since the FDA began reporting public recalls, withdrawals and safety alerts on their website in 2016. Since then, the United States has been significantly impacted by drug recalls affecting angiotensin receptor blockers. This report arms clinicians with additional guidance and provides a framework for responding appropriately to future similar incidents and includes an overview of the angiotensin receptor blockers, and their effects and safety profiles.

Methods This report includes a review of data from all pertinent clinical and scientific sources including information from the FDA's inspection documents and recall website. Additional information is provided on the specific bottles including all lot numbers, expiration dates, etc.

Results The recalls/withdrawals are attributable to the presence of cancer-causing contaminants identified during the manufacturing process from drug manufacturers abroad. The root causes behind the recalls and subsequent shortage appear multifactorial, and stem to a certain extent from the outsourcing of medication manufacturing overseas and lack of quality checks and appropriate oversight.

Conclusions This inherent issue is not likely to resolve soon and has eroded the public trust of/in the healthcare system and the pharmaceutical industry. Patients and healthcare providers are significantly affected and should have a full understanding of the matter in order to guide appropriate response and actions.
\end{abstract}

Keywords Cardiovascular $\cdot$ Angiotensin $\cdot$ FDA $\cdot$ Recall $\cdot$ Drug $\cdot$ Medication

\section{Introduction}

Over the preceding 12-24 months, healthcare providers have encountered an alarming number of recalls related to cardiovascular medications, particularly with angiotensin receptor blockers (ARBs). At least 17 warnings to date have been listed on the Food and Drug Administration recall website (https:// www.fda.gov/safety/recalls-market-withdrawals-safety-

Electronic supplementary material The online version of this article (https://doi.org/10.1007/s10557-020-06976-0) contains supplementary material, which is available to authorized users.

Hani Jneid

Jneid@bcm.edu

1 Section of Cardiology, Baylor College of Medicine and the Michael DeBakey VA Medical Center, 2002 Holcombe Blvd (111), Houston, TX 77030, USA alerts). ARBs, such as valsartan and losartan, represent a class of medications that in randomized controlled clinical trials (RCTs) have been shown to reduce blood pressure (BP) in hypertensive patients and impart cardiovascular benefits in diabetic nephropathy, systolic heart failure, left ventricular dysfunction, and following stroke $[1-8,10]$. They are often prescribed for those individuals who develop a persistent dry cough when taking angiotensin converting enzyme inhibitors (ACE-Is), an adverse event reported to be as prevalent as $35 \%$ in some ethnic groups $[5,6]$.

The first ARB, losartan, was approved by the FDA in 1995, followed by valsartan in 1996, and since then 7 additional ARBs (Table 1) have received approval for clinical use [7]. The most recent estimates from 2013 to 2016 estimates that there are over 116 million adults in the USA with a systolic blood pressure of $130 \mathrm{~mm} \mathrm{Hg}(\mathrm{mmHg})$ or greater [9]. Uncontrolled hypertension can obviously result in major adverse cardiovascular (i.e. stroke, myocardial infarction) events 


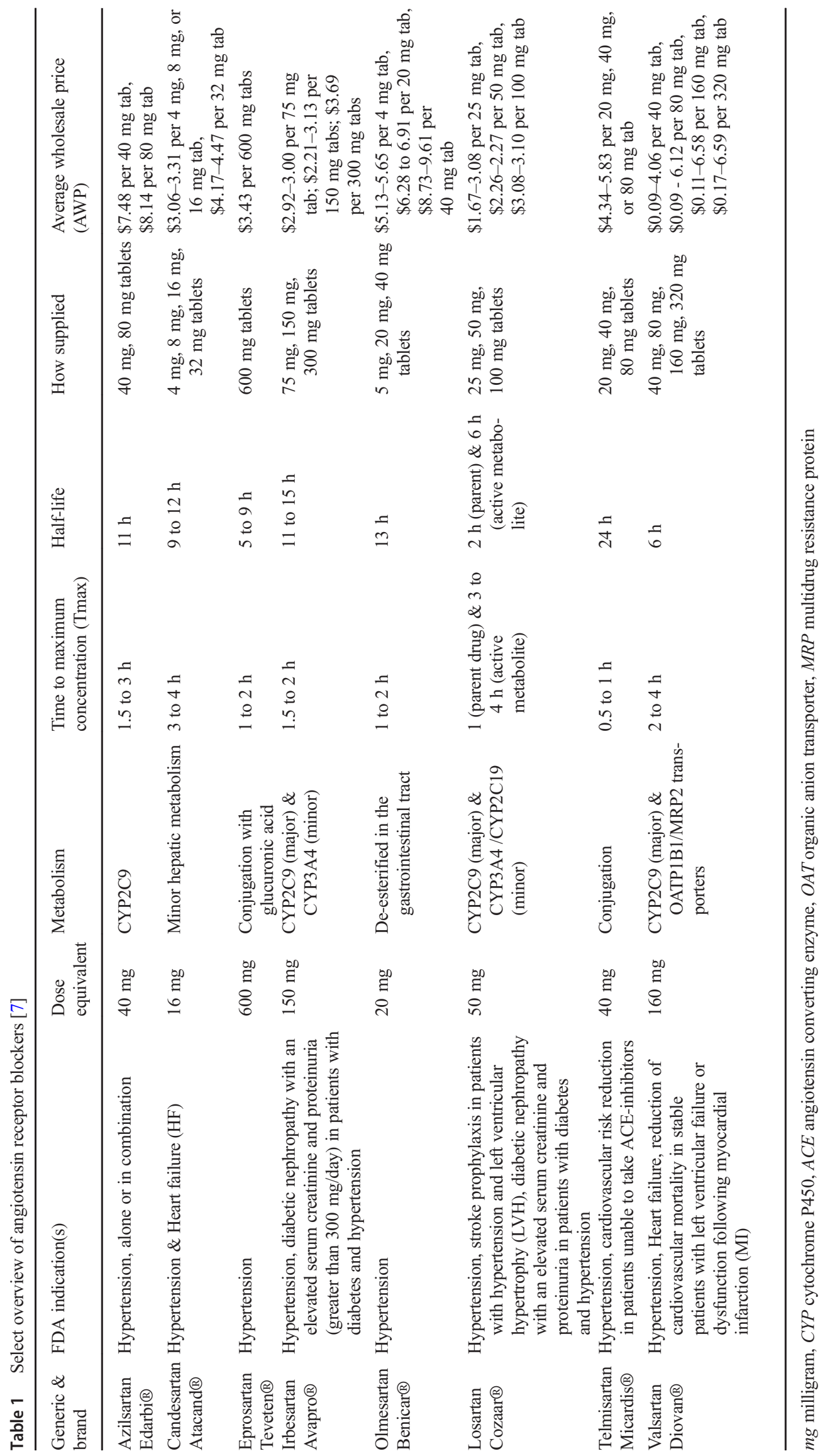


[5-8]. Diabetic nephropathy may also progress to kidney failure and end stage renal disease if not appropriately treated. Given their established benefits, the use of ARBs to reduce cardiovascular morbidity and mortality is likely to further increase since the prevalence of heart failure alone is projected to grow by as much as $46 \%$ from 2012 to 2030 , resulting in well over 8 million affected adults $[9,10]$. Given the prevalence of a myriad of conditions treated with ARBs, it is no surprise that valsartan was one of the top 10 prescribed medications in 2014 with sales grossing over 2 billion dollars per year at that time. Losartan was then listed among the top 10 most widely prescribed drugs with nearly 60 million prescriptions a couple years later [11].

In the current report, we discuss the Food and Drug Administration (FDA) recall of the ARBs and provide an overview of the problem, its intricacies and root causes, and its implication on healthcare in the USA.

\section{Recall Timeline and Affected Lots}

Beginning in July of 2018, the FDA issued a voluntary recall regarding the presence of a contaminant found in the manufacture of valsartan $[12,13]$. What would ensue has become a largely unprecedented sequence of alarming events since the FDA began reporting public recalls, withdrawals, and safety alerts on their website (https://www. fda.gov/Safety/Recalls/default.htm). Since the first recall, the FDA has issued at least 31 additional recalls (Supplement 1) for ARBs specifically valsartan, irbesartan, and losartan due to the presence of at least 3 contaminants, N-Nitroso N-Methyl 4-amino butyric acid (NMBA), N-nitrosodimethyl (NDMA), or Nnitrosodiethylamine (NDEA). It is not unexpected that these 3 individual ARBs were primarily affected as they were the first to gain FDA approval and thus go off patent with valsartan and losartan previously carrying large market share and profits. There have been no other major recalls affecting cardiovascular medications due to carcinogenic contaminants since 2016. This voluntary recall, unfortunately, has impacted several manufacturers including major pharmaceutical companies and was traced back to factories overseas $[12,13]$. The contaminants found within the bottles were alarmingly categorized by the International Agency for Research on Cancer (IARC) and National Toxicology Program 14th Report on Carcinogens as "Group 2A: Probably carcinogenic to humans" and "Reasonably anticipated to be human carcinogens," respectively $[14,15]$. Group $2 \mathrm{~A}$ is listed underneath the IARC's highest classification (i.e. Group 1) for carcinogenic compounds [14]. The FDA previously proposed that the presence of this substance was suspected to be due to changes in how the active chemical agent was manufactured. The production of angiotensin receptor blockers can be quite complex and involves various substitutable ingredients or solvents to make the final product. For instance, the manufacture of valsartan can be completed through at least 4 methods involving an assortment of steps and chemical compounds. The patent report for valsartan alone included approximately 40 pages (https://patents.google.com/patent/WO2008135762A1/ en). However, unskillful production may result in hazardous/flammable byproducts, oily intermediates, which are difficult to crystallize, and can further result in difficulties with purification and/or result in undesirable byproducts. Other techniques may require extensive time and/or resources, which makes production less profitable. It is thus evident that manufacturers including those abroad should undergo intense scrutiny in their design and production of pharmaceutical compounds, but this is not readily done in many countries. Notably, not all ARBs and lots were affected, although the persistence of these two unique compounds found in manufacturing resulted in a high-profile review and delays in production, which ultimately contributed to a shortage of valsartan [15].

\section{Cause of Recall Discovered}

The source of the cancer-causing contaminant was subsequently discovered by the FDA and linked to a chemical byproduct produced during the manufacturing process of the active ingredient from factories overseas [15]. The FDA statement specifically states "specific chemicals and reaction conditions are present.... and may also result from the reuse of materials, such as solvents" [15]. This may not be surprising based on the variability in steps and solvents used to produce chemical compounds but reinforces the need for careful selection and oversight. The FDA also indicated that patients could have been exposed over at least the past 4 years after manufacturers made a change in how they produced the active ingredients. The switch in the manufacturing process may explain why the signal was not detected during preclinical drug development studies and before other companies began manufacturing after patent expiration. Additionally, there was no way of capturing this mishap during routine inspections until recently when scientists became more knowledgeable about unintentional chemical compounds created during manufacturing. The FDA was also unaware how many patients could have been exposed but estimated that up to 2 million people may have been exposed to medications containing carcinogenic impurities. They also speculated that one additional case of cancer might result if 8000 people took the highest dose of an affected medication lot over 4 years (anticipated time that the affected products have been on the market) [15]. 


\section{Guidance to Consumers and Healthcare Providers}

It is extremely important to note again that not all batches were affected. In the FDA recall announcement, patients were advised to check with their pharmacies and healthcare providers first and to not stop their medication unless specifically instructed to do so. This is particularly important given the recent outbreak of the coronavirus disease 2019 (COVID19) and speculation that ACE-Is or ARBs could increase the risk of infection through upregulation of angiotensin converting enzyme-2 receptors (ACE2) thereby leading to inappropriate discontinuation by patients or providers [16]. Given that many of the medications affected were distributed nationwide, pharmacies and healthcare facilities were advised to check inventories and halt distribution and dispensing of affected products immediately. Consumers were directed to contact their respective pharmacy or manufacturer's customer support hotline for queries, while some manufacturers have offered a hotline to provide instructions on how to return affected products (see Supplement 1). Consumers may also cross check their respective lot number with the recall lot numbers listed on the FDA website (https://www.fda.gov/ safety/recalls-market-withdrawals-safety-alerts). Adverse reactions or quality problems experienced by patients with use of affected products should be reported online to the FDA MedWatch program through the following link (www. fda.gov/medwatch/report.htm). Alternatively, the reporting form could be downloaded via (www.fda.gov/MedWatch/ getforms.htm) or by calling. Both patient and providers may request and submit the completed form.

\section{Considerations for Healthcare Providers in Response to the FDA Recall}

Providers and pharmacies are ultimately tasked with addressing questions related to medication replacement or alternatives. From a facility/provider practice perspective, the first step is to identify who was affected by the recall within their respective healthcare system and then create a personalized action plan to address appropriate alternatives. Lists of exposed patients can be obtained if the facility has an electronic medical record/prescribing system using informaticians' help and/or by contacting pharmacies. Although exposure could have been taken place over the past 4 years, the lists should begin by narrowing down to those who were specifically prescribed irbesartan, losartan, and valsartan beginning in July and then cross-checking with the affected lots (Supplement 1 or on the FDA website at https://www.fda.gov/safety/recallsmarket-withdrawals-safety-alerts). Once patients are identified then the decision to replace or provide an alternative should be undertaken. Valsartan is facing a shortage due to the recall, and it is unknown whether additional recalls/shortages may occur in the future. Therefore, it is not unreasonable to consider an alternative ARB or other antihypertensive (i.e. ACE-I if no contraindication or previous allergy/adverse event), although telmisartan and candesartan are also available as generic and could therefore be at similar risk theoretically. If the indication is strictly hypertension, then it might be advisable to have the patient follow-up in clinic for repeat measurements to help determine whether regimen or dosage adjustments are needed to conform to the new BP cutoff of less than 130/80 $\mathrm{mmHg}$ according to the more recent American College of Cardiology (ACC)/American Heart Association (AHA) 2017 Guideline [8]. ACE-Is provide similar BP lowering effects and may be a reasonable alternative to ARBs in the absence of prior intolerance. Table 1 is provided to help identify other alternative ARBs based on FDA-approved indications and clinical characteristics. It is imperative that the provider pay special attention to the indication when determining an alternative, prior allergies/adverse reactions, current medication/ antihypertensive regimen, vital signs, and other considerations (i.e. cost/formulary alternatives, insurance coverage, dosing schedule, potential for noncompliance). Other antihypertensives may be considered with a preference for those with direct evidence of clinical benefits based on the indication and comorbidities with careful consideration given to prior allergies/adverse reactions, lack of contraindications, laboratory findings, vitals, drug interactions, patient preference (i.e. shared decision making), etc. Regardless, all patients should be notified of the recall through letters and/or other forms of communication, particularly for facilities in less informed areas such as rural towns/communities.

\section{Overview of Published Literature of Cancer Link with ARBs}

It is noteworthy that concerns with ARBs causing cancer have dated as far back to 2003 in the effects of candesartan on mortality and morbidity in patients with chronic heart failure (CHARM) trial, which reported a surprising increase in cancer deaths with candesartan versus placebo $(2.3 \%$ vs. $1.6 \% ; p=$ 0.038 ) [17]. Additionally, a slight numerical but nonstatistically significant increase in cancers was shown with losartan in the LIFE ( $8 \%$ vs. $7 \% ; p=0.118$ ) and telmisartan in the TRANSCEND ( $8 \%$ vs. $6.9 \%$, RR $1.17,95 \%$ CI $0.97-$ $1.42 ; p=0.094)$ as well as ONTARGET trials (RR 1.04 $[0.77,1.40)$, respectively $[1,18,19]$. Following these findings, a meta-analysis published by Sipahi and colleagues in 2010 evaluating different solid organ cancers in patients receiving ARBs compared with controls found a significantly higher incidence $(7.2 \%$ vs $6 \%$, risk ratio [RR] $1.08,95 \% \mathrm{CI}$ $1.01-1.15 ; p=0.016)$ particularly with lung cancer 
occurrence [20]. However, subsequent analyses have failed to confirm this association including a more recent comprehensive meta-analysis by Bangalore and colleagues that involved at least 70 randomized controlled trials [21-25]. Interestingly, Bangalor and colleagues did report a slight increase in cancer when ARBs were combined with ACE-Is (2.3\% vs. $2 \%$; RR $1.14,95 \%$ CI 1.02-1.28), but fortunately, combination therapy with ACE-Is is generally not recommended due to lack of efficacy and risk for other harms [25]. Lastly, a meta-analysis was performed by the FDA capturing 31 trials with 84,461 patients on ARBs and 71,355 patients randomized to nonARB comparators with an average follow-up of 39 months. This ultimately ended concerns after reporting incident cancer events of 1.82 per 100 patient-years in the ARB group vs. 1.84 per 100-patient years in the non-ARB group (RR 0.99, 95\% CI 0.92-1.06) [26]. There was no association found between ARBs and cancer-related death (RR 1.04, 95\% CI 0.96-1.13), breast cancer (OR 1.06, 95\% CI 0.9-1.23), lung cancer (OR $1.07,95 \%$ CI $0.89-1.29$ ), or prostate cancer (OR 1.05, (95\% CI $0.95-1.17$ ) and eventually lead the FDA to propose guidance (https://www.fda.gov/regulatory-information/searchfda-guidance-documents/meta-analyses-randomizedcontrolled-clinical-trials-evaluate-safety-human-drugs-orbiological) for conducting meta-analysis when evaluating safety of medications [26]. Previous meta-analyses were most likely affected by significant methodological flaws and uncertainty including heterogeneity in the trial conduct/selection. It should be stated that the trials captured in the FDA's metaanalysis were undertaken decades before the recent findings of differences in ARB manufacturing that exposed patients to carcinogens.

In vitro data have demonstrated expression of the RAS and $\mathrm{AT}_{1 / 2}$ receptors in various cancer cells/tissues including brain, lung, breast, prostate, and pancreatic cancers, and the use of ACE-Is and ARBs were shown to reduce the number of metastases, tumor growth, and vascularization in rodents [27-29]. Although there is a link between AT receptor activation and inflammation, angiogenesis, and cell proliferation, no plausible biologic mechanism has been identified to connect antagonism of AT receptors and cancer development. Moreover, if cancer risk is indeed associated with $\mathrm{AT}_{2}$ activation indirectly through $\mathrm{AT}_{1}$ antagonism, then the cancer risk should be higher with ARBs such as valsartan, olmesartan, azilsartan, and candesartan due to their much higher affinity for the $\mathrm{AT}_{1}$ receptor [27-29]. In summary, concerns regarding a cancer association have not been definitively established and is largely refuted in the healthcare community.

\section{Conclusions}

Although other ARBs were not affected by the recall such as azilsartan, candesartan, eprosartan, olmesartan, and telmisartan, it is possible that other generic ARBs could be affected. Overall, the concerns of cancer and public outcry has been overwhelming to both patients and providers. The angiotensin receptor neprilysin inhibitor (ARNI), valsartan/ sacubitril, remains under patent protection and is therefore unaffected by the recall. Until the etiology of this recall is fully addressed, concerns over prescribing and utilizing ARBs will persist. Because of previous concerns over drug shortages and the potential implications on patient outcomes and healthcare wastage, organizations such as the American Society of Health System Pharmacists (ASHP) have issued a congressional call to action. It is imperative that providers and organizations continue to reach out to members of the congress to take immediate action and further investigate production and manufacturing processes both locally and abroad [30]. Given the global impact of cardiovascular diseases and the use of ARBs for their medical management, it seems prudent to resort to ARBs when ACE-Is cannot be tolerated or when ARBs have proven indications. When doing so, ARBs not affected by the recall should be utilized or other contemporary agents such as valsartan/sacubitril should be considered when clinically appropriate, particularly among heart failure patients with reduced ejection fraction [10].

Authors' Contributions All authors had access to this manuscript and data and played a role in writing this report.

\section{Compliance with Ethical Standards}

Conflicts of Interest The authors declare that they have no conflict of interest.

Ethical Approval This article does not contain any studies with human participants or animals performed by any of the authors.

\section{References}

1. Dahlöf B, Devereux RB, Kjeldsen SE, Julius S, Beevers G, de Faire $\mathrm{U}$, et al. Cardiovascular morbidity and mortality in the Losartan Intervention For Endpoint reduction in hypertension study (LIFE): a randomised trial against atenolol. Lancet. 2002;359: 995-1003.

2. Lithell H, Hansson L, Skoog I, Elmfeldt D, Hofman A, Olofsson B, et al. The Study on Cognition and Prognosis in the Elderly (SCOPE): principal results of a randomized double-blind intervention trial. J Hypertens. 2003;21:875-86.

3. Probstfield JL, O'Brien KD. Progression of cardiovascular damage: the role of renin-angiotensin system blockade. Am J Cardiol. 2010;105(1 Suppl):10A-20A.

4. McFarlane SI, Kumar A, Sowers JR. Mechanisms by which angiotensin-converting enzyme inhibitors prevent diabetes and cardiovascular disease. Am J Cardiol. 2003;91:30H-7H.

5. Dicpinigaitis PV. Angiotensin-converting enzyme inhibitorinduced cough: ACCP evidence-based clinical practice guidelines. Chest. 2006;129(1 Suppl):169S-73S. https://doi.org/10.1378/ chest.129.1_suppl.169S. 
6. Taler SJ. Initial treatment of hypertension. N Engl J Med. 2018;378: 636-44.

7. Gold Standard, Inc. Overview of angiotensin receptor blockers. Clinical Pharmacology [database online]. Available at: http:// www.clinicalpharmacology.com. Accessed: January 26, 2018.

8. ACC/AHA/AAPA/ABC/ACPM/AGS/APhA/ASH/ASPC/NMA/ PCNA Guideline for the prevention, detection, evaluation, and management of high BP in adults: a report of the American College of Cardiology/American Heart Association Task Force on Clinical Practice Guidelines. J Am Coll Cardiol. 2018;71:e127248.

9. Benjamin EJ, Muntner P, Alonso A, Bittencourt MS, Callaway CW, Carson AP, et al. Heart disease and stroke statistics - 2019 update: a report from the American Heart Association. Circulation. 2019 Jan 31;139:e56-528. https://doi.org/10.1161/CIR. 0000000000000659

10. Yancy CW, Jessup M, Bozkurt B, Butler J, Casey de Jr, Colvin MM, et al. 2017 ACC/AHA/HFSA focused updated of the 2013 ACCF/AHA guideline for the management of heart failure: a report of the American College of Cardiology/American Heart Association Task Force on Clinical Practice Guidelines and the Heart Failure Society of America. Circulation. 2017;136(6):e13761.

11. Brooks, M. Top 100 prescribed, top-selling drugs January 12, 2019. https://www.medscape.com/viewarticle/825053

12. BP Charles S. FDA warns about BP medication shortages amid recalls. January 30, 2019. https://www.msn.com/en-us/health/ health-news/fda-warns-about-blood-pressure-medicationshortages-amid-recalls/ar-BBSQQ1q?li=BBnb7Kz

13. Flowers K. 2019, January 26. FDA warns of common BP medicine shortage due to recalls. CNN. Retrieved from https://www.cnn. com/2019/01/26/health/fda-warning-shortage-valsaratn-recalls/ index.html

14. American Cancer Society. Known and probably human carcinogens. January 13, 2019. < https://www.cancer.org/cancer/cancercauses/general-info/known-and-probable-human-carcinogens. $\mathrm{html}>$

15. BP Johnson, CY. 2019, January 25. FDA identifies contamination source in BP medicines used by millions. Washington Post. Retrieved from https://www.washingtonpost.com/health/2019/01/ $25 /$ cancer-causing-contaminant-went-undetected-years-widelyused-blood-pressure-medicines/?noredirect=on

16. Heart Failure Society of America/American College of Cardiology /American Heart Association statement addresses concerns re: using RAAS antagonists in COVID-19. Retrieved from: https:// professional.heart.org/professional/ScienceNews/UCM_505836_ HFSAACCAHA-statement-addresses-concerns-re-using-RAASantagonists-in-COVID-19.jsp

17. Pfeffer MA, Swedberg K, Granger CB, Held P, McMurray J, Michelson EL, et al. Effects of candesartan on mortality and morbidity in patients with chronic heart failure: the CHARMOverall programme. Lancet. 2003;362(9386):759-66.

18. Yusuf S, Sleight P, Anderson C, on behalf of the TRANSCEND Investigators, et al. Effects of the angiotensin-receptor blocker telmisartan on cardiovascular events in high-risk patients intolerant to angiotensin-converting enzyme inhibitors: a randomized controlled trial. Lancet. 2008;372(9644):1174-83.

19. Yusuf S, Koon K, Pogue J, on behalf of the ONTARGET investigators, et al. Telmisartan, ramipril, or both in patients at high risk for vascular events. N Engl J Med. 2008;358:1547-59.

20. Sipahi I, Debanne SM, Rowland DY, Simon DI, Fang JC. Angiotensin-receptor blockade and risk of cancer: meta-analysis of randomized controlled trials. Lancet Oncol. 2010;11:627-36.

21. Connolly S, Yusuf S, Swedberg K, et al On behalf of the ARB Trialists collaboration. Effects of telmisartan, irbesartan, valsartan, candesartan, and losartan on cancers in 15 trials enrolling 138,769 individuals. J Hypertens 2011; 29(4):623-635.

22. Pasternak B, Svanstrom H, Callreus T, et al. Use of angiotensin receptor blockers and the risk of cancer. Circulation. 2011;123: 1729-36.

23. Tascilar K, Azoulay L, Dell'Aniello S, et al. The use of telmisartan and the incidence of cancer. Am J Hypertens. 2016;29(12):135865.

24. Cao L, Zhang S, He W, et al. Antihypertensive drug use and the risk of prostate cancer: a meta-analysis of 21 observational studies.

25. Bangalore S, Kumar S, Kjelsen SE, et al. Antihypertensive drugs and risk of cancer: network meta-analyses and trial sequential analyses of 324,168 participants from randomized trials. Lancet Oncol. 2011;12:65-82.

26. U.S. Food and Drug Administration. 2010, July 15. FDA drug safety communication: no increase in risk of cancer with certain BP drugs - angiotensin receptor blockers (ARBs). Available online at: https://www.fda.gov/Drugs/DrugSafety/ucm257516.htm\# references

27. Deshayes F, Nahmias C. Angiotensin receptors: a new role in cancer? Trends Endocrinol Metab. 2005;16:293-9.

28. Walther T, Menrad A, Orzechowski HD, et al. Differential regulation of in vivo angiogenesis by angiotensin II receptors. FASEBJ. 2003;17:2061-7.

29. Miura S, Karnik SS, Saku K. Review: angiotensin II type 1 receptor blockers: class effects versus molecular effects. J ReninAngiotensin-Aldosterone Syst. 2011;12:1-7.

30. Abramowitz, P. Drug shortages harm patients. January 13, 2019. https://connect.ashp.org/blogs/paul-abramowitz/2018/01/08/drugshortages-harm-patients?ssopc $=1$

Publisher's Note Springer Nature remains neutral with regard to jurisdictional claims in published maps and institutional affiliations. 